\title{
ON JANOWSKI CLOSE-TO-CONVEX FUNCTIONS ASSOCIATED WITH CONIC REGIONS
}

\author{
AFIS SALIU*, KHALIDA INAYAT NOOR \\ Department of Mathematics, COMSATS University Islamabad, Park Road, Tarlai Kalan, Islamabad 45550 , \\ Pakistan \\ *Corresponding author: saliugsu@gmail.com

\begin{abstract}
In this work, we introduce and investigate a class of analytic functions which is a subclass of close-to-convex functions of Janowski type and related to conic regions. Length of the image curve $|z|=r<1$ under the generalized Janowski close-to-convex function is derived. Furthermore, rate of growth of coefficients and Hankel determinant for this class are obtained. Relevant connections of our results with the earlier known results are also pointed out.
\end{abstract}

\section{INTRODUCTION}

Let $E=\{z:|z|<1\}$ and $H$ be the class of functions $f(z)$ defined as

$$
f(z)=z+\sum_{n=2}^{\infty} a_{n} z^{n}
$$

which are analytic in $E$. A function $f(z)$ is subordinate to another function $g(z)$ (written as $f(z) \prec g(z)$ ) if there exists an analytic function $w(z)$ in $E$ with $w(0)=1$ and $|w(z)|<1$ for $z \in E$ such that $f(z)=g(w(z))$.

Let $P_{m}(\alpha)$ be the class of analytic functions $p(z)$ in $E$ satisfying the condition $p(0)=1$ and

$$
\int_{0}^{2 \pi}\left|\frac{\operatorname{Re} p(z)-\alpha}{1-\alpha}\right| d \theta \leq m \pi
$$

Received March $5^{\text {th }}, 2020$; accepted April 13 ${ }^{\text {th }}, 2020$; published May $14^{\text {th }}, 2020$.

2010 Mathematics Subject Classification. 30C45, 30C50, 30C55.

Key words and phrases. analytic functions; open unit disk; Janowski functions; subordination; univalent functions; conic domains.

(C)2020 Authors retain the copyrights of their papers, and all open access articles are distributed under the terms of the Creative Commons Attribution License. 
where $m \geq 2, z=r e^{i \theta}, 0 \leq \alpha<1$, see [12]. The case $\alpha=0$ gives the class $P_{m}$ introduced by Pinchuk [13]. For $\alpha=0, m=2$, we obtain the well-known class $P$ of Carathéodory functions and for $m=2, P_{2}(\alpha) \equiv P(\alpha)$ is the class of functions whose real parts are greater than $\alpha$. It is known in [12] that $p \in P_{m}(\alpha)$ has the integral representation

$$
p(z)=\frac{1}{2 \pi} \int_{0}^{2 \pi} \frac{1+(1-2 \alpha) z e^{-i t}}{1-z e^{-i t}} d v(t),
$$

where $v(t)$ is a function of bounded variation on $[0,2 \pi]$ such that

$$
\int_{0}^{2 \pi} d v(t)=2 \pi \quad \text { and } \quad \int_{0}^{2 \pi}|d v(t)| \leq m \pi
$$

It is seen from (1.3) and (1.4) that $p \in P_{m}(\alpha)$ has a representation

$$
p(z)=\frac{m+2}{4} p_{1}(z)-\frac{m-2}{4} p_{2}(z),
$$

where $p_{i} \in P(\alpha)$ for $i=1,2$.

Denote by $C V, S^{*}, K, C V(\alpha), S^{*}(\alpha), K(\alpha)$, are the subclasses of $S$ (the class of univalent functions in $E$ ) which consist of functions that are convex, starlike, close-to-convex, convex of order $\alpha$, starlike of order $\alpha$ and close-to-convex of order $\alpha(0 \leq \alpha<1)$ respectively. We have the following class of analytic functions in $E$ :

$$
V_{m}(\alpha)=\left\{f \in H: \frac{\left(z f^{\prime}\right)^{\prime}}{f^{\prime}} \in P_{m}(\alpha), z \in E, m \geq 2,0 \leq \alpha<1\right\}, \text { see [12] }
$$

and note that $V_{2}(\alpha) \equiv C V(\alpha)$ and $V_{2}(0) \equiv C V$.

Recently, Noor [11] extended the conic domain $\Omega_{k}, k \geq 0$ introduced by Kanas and Wisniowska [2,3] to that of Janowski type, $\Omega_{k}[A, B],-1 \leq B<A \leq 1$ and defined it as

$$
\begin{aligned}
\Omega_{k}[A, B] & =\left\{u+i v=\left[\left(B^{2}-1\right)\left(u^{2}+v^{2}\right)-2(A B-1) u+\left(A^{2}-1\right)\right]^{2}\right. \\
& \left.>k^{2}\left[\left(-2(B+1)\left(u^{2}+v^{2}\right)+2(A+B+2) u-2(A+1)\right)^{2}+4(A-B)^{2} v^{2}\right]\right\} .
\end{aligned}
$$

Denoted by $k-P(A, B)$, the class of functions $p(z)$ that map $E$ onto $\Omega_{k}[A, B]$. Equivalently, we say $p \in k-P(A, B)$ if and only if

$$
p(z) \prec \frac{(A+1) p_{k}(z)-(A-1)}{(B+1) p_{k}(z)-(B-1)}, k \geq 0,-1 \leq B<A \leq 1,
$$

where the definition of $p_{k}$ is given in [2]. Also, it is worthy mentioning that $p \in k-P(A, B) \subset P\left(\gamma_{1}\right)$ which implies that $p(z)=\left(1-\gamma_{1}\right) h_{1}(z)+\gamma_{1}$, (see [11]) where $h_{1} \in P$ and $\gamma_{1}$ is given by

$$
\gamma_{1}=\frac{2 k+1-A}{2 k+1-B}
$$

If in (1.5), $p_{1}, p_{2} \in k-P(A, B)$, we say $p \in k-P_{m}(A, B)$ and if $P_{m}(\alpha)$ in (1.6) is replaced with $k-P_{m}(A, B)$, we say $f$ belongs to the class $k-U V_{m}(A, B)$. We note that $k-P_{m}(A, B) \subset P_{m}\left(\gamma_{1}\right)$, where $\gamma_{1}$ is given by (1.9). Thus,

$k-U V_{m}(A, B) \subset V_{m}\left(\gamma_{1}\right)$. 
We introduce the following class of functions.

Definition 1.1. Let $f \in H,-1 \leq B<A \leq 1,-1 \leq D<C \leq 1, k \geq 0$ and $m_{1}, m_{2} \geq 2$. Then $f \in k-H_{m_{1} m_{2}}(A, B, C, D)$ if there exists $g \in k-U V_{m_{2}}(C, D)$ such that $\frac{f^{\prime}(z)}{g^{\prime}(z)} \in k-P_{m_{1}}(A, B)$.

In particular,

(i) for $k=0, m_{1}=m=m_{2}, A=1, B=-1, C=1-2 \alpha, D=-1, k-H_{m_{1} m_{2}}(1,-1,1-2 \alpha,-1) \equiv$ $H_{m m}(\alpha)$ is the class of analytic functions studied by Noor [9],

(ii) for $k=0, m_{1}=m=m_{2}, A=1, B=-1, C=1, D=-1$, $k-H_{m_{1} m_{2}}(1,-1,1,-1) \equiv K_{m m}$ is the class of analytic functions investigated by Noor [8],

(iii) for $k=0, m_{1}=2=m_{2}, A=1, B=-1, C=1, D=-1$, $k-H_{22}(1,-1,1,-1) \equiv K$ is the class of close to convex functions first introduced and examined by Kaplan [4]

(iv) for $\left.k=0, m_{1}=2=m_{2}, H_{22}(A, B, C, D) \equiv k-U K(A, B, C, D)\right)$ is the class of analytic functions examined by Mahmood et al [5].

We note that $k-H_{m_{1} m_{2}}(A, B, 1,-1) \equiv H_{m_{1} m_{2}}\left(\gamma_{1}, \sigma\right)$, where $\sigma=\frac{k}{k+1}$.

\section{Some Preliminary Lemmas}

We need the following lemmas to investigate our results.

Lemma 2.1. [10] let $p \in P_{m}(\gamma), 0 \leq \gamma<1, m \geq 2$. Then for $z=r e^{i \theta}$,

$$
\frac{1}{2 \pi} \int_{0}^{2 \pi}|p(z)|^{2} d \theta \leq \frac{1+\left(m^{2}(1-\gamma)^{2}-1\right) r^{2}}{1-r^{2}}
$$

(ii)

$$
\frac{1}{2 \pi} \int_{0}^{2 \pi}\left|p^{\prime}(z)\right| d \theta \leq \frac{m(1-\gamma)}{1-r^{2}}
$$

Lemma 2.2. [12]

(i) $f \in V_{m}(\alpha)$ if and only if there exist $f_{1}, f_{2} \in S^{*}$ such that

$$
f^{\prime}(z)=\frac{\left(\frac{f_{1}(z)}{z}\right)^{\left(\frac{m+2}{4}\right)(1-\alpha)}}{\left(\frac{f_{2}(z)}{z}\right)^{\left(\frac{m-2}{4}\right)(1-\alpha)}} .
$$

(ii) Let $f \in V_{m}(\alpha)$. Then

$$
r\left(\frac{(1-r)^{\left(\frac{m-2}{4}\right)}}{(1+r)^{\left(\frac{m+2}{4}\right)}}\right)^{(1-\alpha)} \leq\left|z f^{\prime}(z)\right| \leq r\left(\frac{(1+r)^{\left(\frac{m-2}{4}\right)}}{(1-r)^{\left(\frac{m+2}{4}\right)}}\right)^{(1-\alpha)}
$$


We will need the hypergeometric function

$$
\frac{\Gamma(a) \Gamma(c-a)}{\Gamma(c)} G(a, b, c ; z)=\int_{0}^{1} u^{a-1}(1-u)^{c-a-1}(1-z u)^{-b} d u .
$$

Unless otherwise stated, we assume, $m_{1}, m_{2} \geq 2, k \geq 0-1 \leq B<A \leq 1$, and $-1 \leq D<C \leq 1$

\section{Main Results}

Theorem 3.1. Let $f \in k-H_{m_{1} m_{2}}(A, B, C, D)$. Then for $0<r<1$,

$$
\begin{array}{r}
L(r, f) \leq \pi\left\{\mathfrak{C}\left(m_{2}, k, \gamma_{2}, C, D\right) M(r) \log \frac{1}{1-r}+\right. \\
+\frac{2^{b+1} \gamma_{1}}{a}[[G(a, b, c,-1)-2 G(a, 1+b, c-1)] \\
\left.\left.+r_{1}^{a}\left[2 G\left(a, 1+b, c,-r_{1}\right)-G\left(a, b, c,-r_{1}\right)\right]\right]\right\}
\end{array}
$$

where $M(r)=\max _{\theta}\left|f\left(r e^{i \theta}\right)\right|, \mathfrak{C}\left(m_{2}, k, \gamma_{2}, C, D\right)$ is a constant depending on $m_{2}, k, \gamma_{2}, C$ and $D$,

$$
a=\left(\frac{m_{2}}{2}-1\right)\left(1-\gamma_{2}\right), \quad b=2\left(\gamma_{2}-1\right), c=a+1 \text { and } r_{1}=\frac{1-r}{1+r}
$$

where

$$
\gamma_{1}=\frac{2 k+1-A}{2 k+1-B}, \quad \gamma_{2}=\frac{2 k+1-C}{2 k+1-D}
$$

Proof. Let $z=r e^{i \theta}$. Then

$$
\begin{aligned}
L(r, f) & =\int_{0}^{2 \pi}\left|z f^{\prime}(z)\right| d \theta \\
& =\int_{0}^{2 \pi}\left|z g^{\prime}(z) p(z)\right| d \theta, \text { where } g \in k-V_{m_{2}}(C, D) \text { and } p \in k-P_{m_{1}}(A, B) \\
& \leq \int_{0}^{r} \int_{0}^{2 \pi}\left(z g^{\prime}(z)\right)^{\prime} p(z)\left|d \theta d \rho+\int_{0}^{r} \int_{0}^{2 \pi}\right| z g^{\prime}(z) p^{\prime}(z) \mid d \theta d \rho \\
& =\mathcal{J}_{1}(r)+\mathcal{J}_{2}(r) .
\end{aligned}
$$

Let

$$
\frac{\left(z g^{\prime}\right)^{\prime}(z)}{g^{\prime}(z)}=\mathcal{H}(z)=1+\sum_{n=1}^{\infty} d_{n} z^{n} \in k-P_{m_{2}}(C, D) .
$$

Then by Schwarz inequality and Perseval's theorem, we have

$$
\begin{aligned}
\mathcal{J}_{1}(r) \leq 2 \pi\left(\int_{0}^{r} \int_{0}^{2 \pi}\left|f^{\prime}(z)\right|^{2} d \theta d \rho\right)^{\frac{1}{2}}\left(\int_{0}^{r} \int_{0}^{2 \pi}|\mathcal{H}(z)|^{2} d \theta d \rho\right)^{\frac{1}{2}} \\
=2 \pi\left(\int_{0}^{r} \sum_{n=1}^{\infty} n^{2}\left|a_{n}\right|^{2} \rho^{2 n-2} d \rho\right)^{\frac{1}{2}}\left(\int_{0}^{r} \sum_{n=0}^{\infty}\left|d_{n}\right|^{2} \rho^{2 n} d \rho\right)^{\frac{1}{2}} .
\end{aligned}
$$


It is easy to see that

$$
\left|d_{n}\right| \leq \frac{m_{2}(C-D)\left|\delta_{k}\right|}{4}
$$

where $\delta_{k}$ has its definition given in [11]. Therefore,

$$
\begin{aligned}
\mathcal{J}_{1}(r) & \leq \frac{\sqrt{2} \pi m_{2}(C-D)\left|\delta_{k}\right|}{4}\left(\frac{1}{r} \sum_{n=1}^{\infty} \frac{n^{2}}{2 n-1}\left|a_{n}\right|^{2} r^{2 n}\right)^{\frac{1}{2}}\left(\log \frac{1+r}{1-r}\right)^{\frac{1}{2}} \\
& \leq \frac{\sqrt{2} \pi m_{2}(C-D)\left|\delta_{k}\right|}{4} M(r)\left(\frac{1}{r} \log \frac{1+r}{1-r}\right)^{\frac{1}{2}},
\end{aligned}
$$

where we used the fact that $A(r)=\pi \sum_{n=1}^{\infty} n\left|a_{n}\right|^{2} r^{2 n}$ is the area of the image of $|z|<r$ bounded by $w=f(z)$ and $A(r) \leq \pi M^{2}(r)$.

Next, we estimate $\mathcal{J}_{2}(r)$. Since $p \in k-P_{m_{1}}(A, B) \subset P_{m_{1}}\left(\gamma_{1}\right)$, then using (1.3), we get

$$
p^{\prime}(z)=\frac{\left(1-\gamma_{1}\right)}{\pi} \int_{0}^{2 \pi} \frac{e^{i t}}{\left(1-z e^{i t}\right)^{2}} d v(t) \quad \text { and } \quad \int_{0}^{2 \pi} \frac{1-\rho^{2}}{\left|1-z e^{i t}\right|^{2}} d v(t)=\frac{2 \pi\left(\operatorname{Rep}(z)-\gamma_{1}\right)}{1-\gamma_{1}}
$$

Therefore,

$$
\begin{aligned}
\mathcal{J}_{2}(r) & \leq \frac{\left(1-\gamma_{1}\right)}{\pi} \int_{0}^{r} \int_{0}^{2 \pi} \int_{0}^{2 \pi} \frac{\left|z g^{\prime}(z)\right|}{\left|1-z e^{-i t}\right|^{2}} d v(t) d \theta d \rho \\
& =2 \int_{0}^{r} \int_{0}^{2 \pi}\left|z g^{\prime}(z)\right|\left(\operatorname{Rep}(z)-\gamma_{1}\right) \frac{1}{1-\rho^{2}} d \theta d \rho \\
& =2 \int_{0}^{r} \int_{0}^{2 \pi} \operatorname{Re}\left(z g^{\prime}(z) e^{-i \arg z g^{\prime}(z)} p(z)\right) \frac{1}{1-\rho^{2}} d \theta d \rho-2 \gamma_{1} \int_{0}^{r} \int_{0}^{2 \pi}\left|z g^{\prime}(z)\right| d \theta d \rho .
\end{aligned}
$$

Integration by parts, application of (1.2) and Lemma 2.2(ii) give

$$
\begin{aligned}
\mathcal{J}_{2}(r) & \leq 2 \pi\left(m_{2}\left(1-\gamma_{2}\right)+2 \gamma_{2}\right) \int_{0}^{r} \frac{M(\rho)}{1-\rho^{2}} d \rho-4 \pi \gamma_{1} \int_{0}^{r} \rho \frac{(1-\rho)^{\left(\frac{m-2}{4}\right)\left(1-\gamma_{2}\right)-1}}{(1+\rho)^{\left(\frac{m+2}{4}\right)\left(1-\gamma_{2}\right)+1}} d \rho \\
& \leq \pi\left(m_{2}\left(1-\gamma_{2}\right)+2 \gamma_{2}\right) M(r) \log \frac{1+r}{1-r}+4 \pi \gamma_{1}\left(L_{1}(r)-L_{2}(r)\right)
\end{aligned}
$$

where

$$
L_{1}(r)=\int_{0}^{r} \frac{(1-\rho)^{\left(\frac{m-2}{4}\right)\left(1-\gamma_{2}\right)-1}}{(1+\rho)^{\left(\frac{m+2}{4}\right)\left(1-\gamma_{2}\right)+1}} d \rho \quad \text { and } \quad L_{2}(r)=\int_{0}^{r} \frac{(1-\rho)^{\left(\frac{m-2}{4}\right)\left(1-\gamma_{2}\right)-1}}{(1+\rho)^{\left(\frac{m+2}{4}\right)\left(1-\gamma_{2}\right)}} d \rho .
$$

Let $u=\frac{1-\rho}{1+\rho}$, so that $d \rho=-\frac{2}{(1+u)^{2}}$. Then

$$
\begin{aligned}
L_{1}(r)= & \left(\frac{1}{2}\right)^{2\left(2-\gamma_{2}\right)-1}\left[\int_{0}^{1} u^{\left(\frac{m_{2}}{2}-1\right)\left(1-\gamma_{2}\right)-1}(1+u)^{2\left(1-\gamma_{2}\right)} d u-\int_{0}^{r_{1}} u^{\left(\frac{m_{2}}{2}-1\right)\left(1-\gamma_{2}\right)-1}(1+u)^{2\left(1-\gamma_{2}\right)} d u\right] \\
& =\frac{1}{a} G(a, b, c,-1)-\int_{0}^{r_{1}} u^{\left(\frac{m_{2}}{2}-1\right)\left(1-\gamma_{2}\right)-1}(1+u)^{2\left(1-\gamma_{2}\right)} d u
\end{aligned}
$$

where $a, b, c$ and $r_{1}$ are given in Theorem 3.1. For the second integral in (3.7), we let $u=r_{1} v$. Then

$$
L_{1}(r)=\frac{2^{b-1}}{a}\left[G(a, b, c,-1)-r_{1}^{a} G\left(a, b, c,-r_{1}\right)\right]
$$


In a similar way, we obtain

$$
L_{2}(r)=\frac{2^{b}}{a}\left[G(a, 1+b, c,-1)-r_{1}^{a} G\left(a, 1+b, c,-r_{1}\right)\right] .
$$

Using (3.8), (3.9) in (3.6), we get

$$
\begin{aligned}
\mathcal{J}_{2}(r) \leq \pi & \left(m_{2}\left(1-\gamma_{2}\right)+2 \gamma_{2}\right) M(r) \log \frac{1+r}{1-r}+\frac{\pi 2^{b+1} \gamma_{1}}{a}\{[G(a, b, c,-1)-2 G(a, 1+b, c-1)] \\
& \left.+r_{1}^{a}\left[2 G\left(a, 1+b, c,-r_{1}\right)-G\left(a, b, c,-r_{1}\right)\right]\right\}
\end{aligned}
$$

The estimates for $\mathcal{J}_{1}(r)$ and $\mathcal{J}_{2}(r)$ yield the required result.

Corollary 3.1. Let $f \in K_{m_{1} m_{2}}$, Then for $0 \leq r<1$,

$$
L(r, f) \leq \mathfrak{C}\left(m_{2}\right) M(r) \log \frac{1}{1-r},
$$

where $M(r)=\max _{\theta}\left|f\left(r e^{i \theta}\right)\right|, \mathfrak{C}\left(m_{2}\right)$ is a constant depending on $m_{2}$.

Corollary 3.2. Let $f \in H_{m m}(\alpha)$, Then for $0 \leq r<1$,

$$
L(r, f) \leq \mathfrak{C}(m, \alpha) M(r) \log \frac{1}{1-r},
$$

where $M(r)=\max _{\theta}\left|f\left(r e^{i \theta}\right)\right|, \mathfrak{C}(m, \alpha)$ is a constant depending on $m$ and $\alpha$.

Corollary 3.3. Let $f \in K$. Then for $0 \leq r<1$,

$$
L(r, f) \leq \mathfrak{C} M(r) \log \frac{1}{1-r}
$$

where $M(r)=\max _{\theta}\left|f\left(r e^{i \theta}\right)\right|, \mathfrak{C}$ is a constant.

Theorem 3.2. Let $f(z)$ be of the form (1.1) and $f \in k-H_{m_{1} m_{2}}(A, B, C, D)$. Then

$$
\begin{aligned}
\left|a_{n}\right| \leq & \frac{\pi}{n}\left(\mathfrak{C}_{1}\left(m_{2}, k, \gamma_{2}, C, D\right) M\left(\frac{n-1}{n}\right) \log n+\frac{2^{b+1} \gamma_{1}}{a}\{[G(a, b, c,-1)-2 G(a, 1+b, c-1)]\right. \\
& \left.\left.+r_{1}^{a}\left[2 G\left(a, 1+b, c,-\frac{1}{2 n-1}\right)-G\left(a, b, c,-\frac{1}{2 n-1}\right)\right]\right\}\right),
\end{aligned}
$$

where $\gamma_{1}, \gamma_{2}, a, b$ and $c$ are given as in Theorem 3.1.

Noonan and Thomas [6] define for $q \geq 1, n \geq 1$, the $q$ th Hankel determinant of $f(z) \in H$ as follows:

$$
\mathcal{H}_{q}(n)=\left|\begin{array}{cccc}
a_{n} & a_{n+1} & \ldots & a_{n+q-1} \\
a_{n+1} & a_{n+2} & \ldots & a_{n+q-2} \\
\vdots & \vdots & \vdots & \vdots \\
a_{n+q-1} & a_{n+q-2} & \ldots & a_{n+2 q-2}
\end{array}\right| .
$$


To estimate the growth rate of Hankel determinant for $f \in k-H_{m_{1} m_{2}}(A, B, C, D)$, we need the following results due to Noonan and Thomas [6].

Lemma 3.1. let $f \in H$ and suppose the qth Hankel determinant of $f(z)$ for $q \geq 1, n \geq 1$ is given by (3.11). Then writing $\Delta_{j}(n)=\Delta_{j}\left(n, z_{1}, f\right)$, we have

$$
\mathcal{H}_{q}(n)=\left|\begin{array}{cccc}
\Delta_{2 q-2}(n) & \Delta_{2 q-3}(n+1) & \ldots & \Delta_{q-1}(n+q-1) \\
\Delta_{2 q-3}(n+1) & \Delta_{2 q-4}(n+2) & \ldots & \Delta_{q-2}(n+q-2) \\
\vdots & \vdots & \vdots & \vdots \\
\Delta_{q-1}(n+q-1) & \Delta_{q-2}(n+q-2) & \ldots & \Delta_{q}(n+2 q-2)
\end{array}\right|,
$$

where with $\Delta_{0}\left(n, z_{1}, F\right)=a_{n}$, we define for $j \geq 1$,

$$
\Delta_{j}\left(n, z_{1}, f\right)=\Delta_{j-1}\left(n, z_{1}, f\right)-z_{1} \Delta_{j-1}\left(n+1, z_{1}, f\right) .
$$

Lemma 3.2. With $x=\left(\frac{n}{n+1}\right) y, u \geq 0$ an integer,

$$
\Delta_{j}\left(n+u, u, x, z f^{\prime}(z)\right)=\sum_{i=0}^{j}\left(\begin{array}{l}
j \\
i
\end{array}\right) \frac{y^{i}(u-(i-1) n)}{(n+1)^{i}} \cdot \Delta_{j-i}(n+u+i, y, f) .
$$

Remark 3.1. Consider any determinant of the form

$$
\mathcal{D}=\left|\begin{array}{cccc}
y_{2 q-2} & y_{2 q-3} & \ldots & y_{q-1} \\
y_{2 q-3} & y_{2 q-4} & \ldots & y_{q-2} \\
\vdots & \vdots & \vdots & \vdots \\
y_{q-1} & y_{q-2} & \cdots & y_{0}
\end{array}\right|
$$

with $1 \leq i, j \leq q$ and $\alpha_{i j}=y_{2 q-(i+j)}, \mathcal{D}=\operatorname{det}\left(\alpha_{i j}\right)$. Thus

$$
\mathcal{D}=\sum_{v_{1} \in S_{q}}\left(\operatorname{sgn} v_{1}\right) \prod_{j=1}^{q}\left(y_{2 q}-\left(v_{1}(j)+j\right)\right.
$$

where $S_{q}$ is the symmetric group on $q$ elements and $\operatorname{sgn} v_{1}$ is either +1 or -1 . Thus, in the expansion of $\mathcal{D}$, each summand has $q$ factor and the sum of the subscripts of the factor of each summand is $q^{2}-q$.

Now let $n$ be given and $\mathcal{H}_{q}(n)$ is as Lemma 3.1, then each summand in the expression of $\mathcal{H}_{q}(n)$ is of the form

$$
\prod_{i=1}^{q} \Delta_{v_{1}(i)}\left(n+2 q-2-v_{1} i\right)
$$

where $v_{1} \in S_{q}$ and

$$
\sum_{i=1}^{q} v_{1}(i)=q^{2}-q ; \quad 0 \leq v_{1}(i) \leq 2 q-2
$$


Theorem 3.3. Let $f \in H_{m_{1} m_{2}}\left(\gamma_{1}, \sigma\right)$ and $\left(m_{2}+2\right)\left(1-\gamma_{2}\right) \geq 4 j$. If the qth Hankel determinant of $f(z)$ for $q \geq 1, n \geq 1$ is given by (3.11), then

$$
\mathcal{H}_{q}(n)=\mathrm{O}(1)\left\{\begin{array}{l}
n^{\left(\frac{m_{2}}{2}+1\right)\left(\frac{1}{k+1}\right)-1}, q=1, \\
n^{\left(\frac{m_{2}}{2}+1\right)\left(\frac{1}{k+1}\right) q-q^{2}}, q \geq 2, m_{2} \geq 8(k+1)(q-1)-2
\end{array},\right.
$$

where $\mathrm{O}(1)$ is a constant that depends on $m_{1}, m_{2}, j, \gamma_{1}, k$ only, with $\gamma_{1}$ given by (3.3).

Proof. Since $f \in H_{m_{1} m_{2}}\left(\gamma_{1}, \sigma\right)$, then

$$
f^{\prime}(z)=p(z) g^{\prime}(z)
$$

where $g^{\prime}(z) \in k-V_{m_{2}}(1,-1) \subset V_{m_{2}}(\sigma)$ and $p(z) \in k-P_{m_{1}}(A, B) \subset P_{m_{1}}\left(\gamma_{1}\right)$. Setting

$$
\mathcal{F}(z)=\left(z f^{\prime}(z)\right)^{\prime}, \quad \text { and } \quad \frac{\left(z g^{\prime}(z)\right)^{\prime}}{g^{\prime}(z)}=h(z)
$$

then

$$
\mathcal{F}(z)=g^{\prime}(z)\left(h(z) p(z)+z p^{\prime}(z)\right)
$$

Now, for $j \geq 0, z_{1}$ any nonzero complex number, consider $\Delta_{j}\left(n, z_{1}, \mathcal{F}(z)\right)$ as defined by (3.13). Then

$$
\begin{aligned}
\Delta_{j}\left(n, z_{1}, \mathcal{F}(z)\right) & \leq \frac{1}{2 \pi r^{n+j}}\left|\int_{0}^{2 \pi}\left(z-z_{1}\right)^{j} \mathcal{F}(z) e^{-i(n+j) \theta} d \theta\right| \\
& \leq \frac{1}{2 \pi r^{n+j}} \int_{0}^{2 \pi}\left|z-z_{1}\right|^{j}\left|g^{\prime}(z) \| h(z) p(z)+z p^{\prime}(z)\right| d \theta .
\end{aligned}
$$

Using Lemma 2.2(i) and the distortion theorems for starlike function, then for $\left(m_{2}+2\right)(1-\sigma) \geq 4 j$, we obtain

$$
\begin{aligned}
\Delta_{j}\left(n, z_{1}, \mathcal{F}(z)\right) \leq & \frac{1}{2 \pi r^{n+j}} \int_{0}^{2 \pi}\left|\left(z-z_{1}\right) f_{1}(z)\right|^{j} \frac{\left|f_{1}(z)\right|^{\left(\frac{m_{2}+2}{4}\right)(1-\sigma)-j}}{\left|f_{2}(z)\right|^{\left(\frac{m_{2}-2}{4}\right)(1-\sigma)}}\left|h(z) p(z)+z p^{\prime}(z)\right| d \theta \\
\leq & \frac{1}{2 \pi r^{n+j-\sigma}} \int_{0}^{2 \pi}\left|\left(z-z_{1}\right) f_{1}(z)\right|^{j}\left(\frac{r}{(1-r)^{2}}\right)^{\left(\frac{m_{2}+2}{4}\right)(1-\sigma)-j}\left(\frac{(1+r)^{2}}{r}\right)^{\left(\frac{m_{2}-2}{4}\right)(1-\sigma)} \\
& \times\left|h(z) p(z)+z p^{\prime}(z)\right| d \theta .
\end{aligned}
$$

Using the result of Golusin [1] and Schwarz inequality, we arrive at

$$
\begin{aligned}
\left|\Delta_{j}\left(n, z_{1}, \mathcal{F}(z)\right)\right| \leq & \frac{2^{\left(\frac{m_{2}-2}{2}\right)(1-\sigma)+j}}{r^{n-1}}\left(\frac{1}{1-r}\right)^{\left(\frac{m_{2}-2}{2}\right)(1-\sigma)-j} \\
& \times\left\{\left(\frac{1}{2 \pi} \int_{0}^{2 \pi}|h(z)|^{2}\right)^{\frac{1}{2}}\left(\frac{1}{2 \pi} \int_{0}^{2 \pi}|p(z)|^{2}\right)^{\frac{1}{2}}+\frac{1}{2 \pi} \int_{0}^{2 \pi}\left|z p^{\prime}(z)\right|\right\} d \theta .
\end{aligned}
$$


In view of Lemma 2.1 , we get

$$
\begin{aligned}
\left|\Delta_{j}\left(n, z_{1}, \mathcal{F}(z)\right)\right| \leq & \frac{2^{\left(\frac{m_{2}-2}{2}\right)(1-\sigma)+j}}{r^{n-1}}\left(\frac{1}{1-r}\right)^{\left(\frac{m_{2}+2}{2}\right)(1-\sigma)-j}\left\{\left[\frac{1+\left(m_{2}^{2}(1-\sigma)^{2}-1\right) r^{2}}{1-r^{2}}\right]^{\frac{1}{2}}\right. \\
& \left.\times\left[\frac{1+\left(m_{1}^{2}\left(1-\gamma_{1}\right)^{2}-1\right) r^{2}}{1-r^{2}}\right]^{\frac{1}{2}}+\frac{r m_{1}\left(1-\gamma_{1}\right)}{1-r^{2}}\right\} \\
\leq & \frac{2^{\left(\frac{m_{2}-2}{2}\right)\left(1-\gamma_{2}\right)+j}\left(m_{1}\left(1-\gamma_{1}\right)+\left(m_{1}^{2}\left(1-\gamma_{1}\right)^{2}+1\right)^{\frac{1}{2}}\left(m_{2}^{2}(1-\sigma)^{2}+1\right)^{\frac{1}{2}}\right)}{r^{n-1}} \\
& \times\left(\frac{1}{1-r}\right)^{\left(\frac{m_{2}-2}{2}\right)(1-\sigma)-j+1} .
\end{aligned}
$$

Applying Lemma 3.2 with $z_{1}=\left(\frac{n}{1+n}\right)^{2} e^{i \theta_{n}},(n \rightarrow \infty), r=1-\frac{1}{n}$, we have for $\left(m_{2}+2\right)\left(1-\gamma_{2}\right) \geq 4 j$,

$$
\Delta_{j}\left(n, e^{i \theta_{n}}, \mathcal{F}(z)\right)=\mathrm{O}(1) n^{\left(\frac{m_{2}+2}{2}\right)(1-\sigma)-j+1},
$$

where $\mathrm{O}(1)$ is a constant that depends on $m_{1}, m_{2}, \gamma_{1}$ and $\sigma$. We estimate the rate of growth of $\mathcal{H}_{q}(n)$ for $f \in H_{m_{1} m_{2}}\left(\gamma_{1}, \sigma\right)$. Then, for $\mathrm{q}=1, \mathcal{H}_{1}(n)=a_{n}=\Delta_{0}(n)$ and

$$
\mathcal{H}_{1}(n)=\mathrm{O}(1) n^{\left(\frac{m_{2}+2}{2}\right)\left(\frac{1}{1+k}\right)-1}
$$

For $q \geq 2$, we use similar arguments from Noonan and Thomas [6] along with Lemma 3.1 and Remark 3.1 to arrive at

$$
\mathcal{H}_{q}(n)=\mathrm{O}(1) n^{\left(\frac{m_{2}+2}{2}\right)\left(\frac{1}{1+k}\right) q-q^{2}}, m_{2} \geq 8(k+1)(q-1)-2
$$

Corollary 3.4. [8] If $f \in K_{m m}$, then

$$
\mathcal{H}_{q}(n)=\mathrm{O}(1)\left\{\begin{array}{l}
n^{\frac{m_{2}}{2}}, q=1, \\
n^{\left(\frac{m_{2}}{2}+1\right) q-q^{2}}, q \geq 2, m_{2} \geq 8(q-1)-2
\end{array},\right.
$$

where $\mathrm{O}(1)$ is a constant that depends on $m$ and $j$, only.

Corollary 3.5. If $f \in 1-H m_{1} m_{2}(A, B, 1,-1)$, then

$$
\mathcal{H}_{q}(n)=\mathrm{O}(1)\left\{\begin{array}{l}
n^{\frac{m_{2}}{4}-\frac{1}{2}}, q=1, \\
n^{\left(\frac{m_{2}}{4}+\frac{1}{2}\right) q-q^{2}}, q \geq 2, m_{2} \geq 16(q-1)-2
\end{array}\right.
$$

where $\mathrm{O}(1)$ is a constant that depends on $\gamma_{1}, m_{1}$ and $j$, only. 


\section{Conclusion}

Arc length and rate of growth of Hankel determinant problems have always been the main interests of many researchers in Geometric function theory. Many studies associated to these problems revolved around classes of normalized analytic univalent functions. In this particular work, length of the image curve $|z|=r<1$ under the generalized Janowski close-to-convex function was proved; rate of growth of coefficients and Hankel determinant for this class were also obtained.

\section{ACKNOWLEDGEMENTS}

The authors would like to thank Prof. Dr. Raheel Qamar, Rector, COMSATS University Islamabad, Pakistan, for providing excellent research and academic environments.

Conflicts of Interest: The author(s) declare that there are no conflicts of interest regarding the publication of this paper.

\section{REFERENCES}

[1] G. Golusin, On distortion theorems and coefficients of univalent functions, Mat. Sb. 19(1946), 183-203.

[2] S. Kanas, A. Wisniowska, Conic regions and k-uniform convexity, J. Comput. Appl. Math. 105(1-2)(1999), 327-336.

[3] S. Kanas, A. Wisniowska, Conic domains and starlike functions, Rev. Roumaine Math. Pures Appl. 45(4)(2000), 647-658.

[4] W. Kaplan, Close-to-convex schlicht functions, Michigan Math. J. 1(2)(1952), 169-185.

[5] S. Mahmood, M. Arif, S. N. Malik, Janowski type close-to-convex functions associated with conic regions, J. Inequal. Appl. 2017(1)(2017), 259.

[6] J. W. Noonan, D. K.Thomas, On the Hankel determinants of areally mean p-valent functions, Proc. Lond. Math. Soc. $3(3)(1972), 503-524$.

[7] K. I. Noor, On a generalization of close-to-convexity, Int. J. Math. Math. Sci. 6(2)(1983), 327-333.

[8] K. I. Noor, On analytic functions related with functions of bounded boundary rotation, Comment. Math. Univ. St. Pauli, 30(2)(1981), 113-118.

[9] K. I. Noor, M. A. Noor, Higher order close-to-convex functions, Math. Japon. 1992.

[10] K. I. Noor, On subclasses of close-to-convex functions of higher order, Int. J. Math. Math. Sci. 15(2)(1992), $279-289$.

[11] K. I. Noor, S. N. Malik, On coefficient inequalities of functions associated with conic domains, Comput. Math. Appl. 62(5) (2011), 2209-2217.

[12] K. S. Padmanabhan, R. Parvatham, Properties of a class of functions with bounded boundary rotation, Ann. Polon. Math. 3(31)(1976), 311-323.

[13] B. Pinchuk, Functions of bounded boundary rotation, Israel J. Math. 10(1)(1971), 6-16.

[14] C. Pommerenke, Uber nahezu konvexe analytische Funktionenber nahezu konvexe analytische Funktionen, Arch. Math. (Basel), 16(1)(1965), 344-347.

[15] D. K. Thomas, On starlike and close-to-convex univalent functions, J. Lond. Math. Soc. 1(1)(1967), 427-435. 